\section{Respiratory Syncytial Virus Outbreak Prevention by Screening Neonates with Respiratory Infection, Isolation and Applying Standard Infection Control Procedures}

\author{
Mehmet Mutlu ${ }^{1 *}$, Ayhan Söğüt ${ }^{2}$, Şebnem Kader ${ }^{1}$ and Yakup \\ Aslan ${ }^{1}$ \\ ${ }^{1}$ Division of Neonatology, Department of Pediatrics, Karadeniz Technical \\ University Faculty of Medicine, Trabzon, Turkey \\ ${ }^{2}$ Department of Pediatric Allergy, Regional Training and Research Hospital, \\ Erzurum, Turkey
}

\begin{abstract}
Objective: To determine the ability of early antigen detection, isolation of infected infants with Respiratory Syncytial Virus (RSV) and applied of standard infection control procedures to prevent nosocomial spread of virus from isolation rooms to the Neonatal Intensive Care Unit (NICU) where high-risk infants were under care. Methods: The study was performed between 1 October 2010 and 31 March 2011, prospectively. All newborns who were admitted to the NICU for Lower Respiratory Tract Infection (LRTI) (Group 1) were first tested using anusing Respi-Strip antigen detection kits to identify RSV (+) neonates. RSV (+) neonates with LRTI (Group 1a) were isolated and segregated in a room separate from RSV (-) neonates with LRTI (Group 1b). Infants at high risk for RSV infection who had been hospitalized continuously since birth (Group 2) were also segregated and observed at least weekly as sentinels for nosocomial spread of RSV infection. Standard infection control procedures were in place throughout the study period.

Findings: Forty-one newborns were hospitalized and treated with a diagnosis of LRTI during the study period. Fifteen (37\%) of these were RSV antigen-positive. No RSV outbreak or infection was observed with isolation and standard infection control procedures in the risk group premature infants during their hospitalization. Also this study show that; RSV related LRTI is high in neonatal period (37\%). Conclusion: This study showed that isolation of infants with RSV and strict adherence to infection control procedures might prevent nosocomial spread of RSV infection in the NICU that care for at risk infants even in the absence of palivizumab prophylaxis.
\end{abstract}

*Corresponding author: Mehmet Mutlu, Division of Neonatology, Department of Pediatrics, Karadeniz Technical University Faculty of Medicine, Trabzon, Turkey, Tel: +90 05326332749; E-mail: drmehmetmutlu38@hotmail.com

Citation: Mutlu M, Söğüt A, Kader Ş, Aslan Y (2015) Respiratory Syncytial Virus Outbreak Prevention by Screening Neonates with Respiratory Infection, Isolation and Applying Standard Infection Control Procedures. J Neonatol Clin Pediatr 2: 006.

Received: January 16, 2015; Accepted: February 14, 2015; Published: March 02, 2015
Keywords: Isolation; Neonatal intensive care unit; Newborn; Outbreak; Respiratory syncytial virus; Standard infection control procedures

Respiratory Syncytial Virus (RSV), RNA virus from the family Paramyxoviridae, is the most frequent cause of Lower Respiratory Tract Infections (LRTI) in infants [1-3]. RSV causes significant LRTI that require hospitalization, especially in newborn [2]. Premature infants (gestational age $<35$ weeks), infants with bronchopulmonary dysplasia or hemodynamically significant congenital heart disease are among those at highest risk for severe RSV illness [1,2]. Nosocomial outbreaks of RSV can occur and have important cost and outcomes [4].

Isolation of the patients with RSV, standard infection control procedures and maintenance of stringent hygienic conditions and/or the administration of palivizumab are important to prevent the spread of the RSV outbreaks in the Neonatal Intensive Care Unit (NICU) [5-14]. However, there is no consensus on the management of outbreaks of nosocomial RSV infection in NICU.

The aim of this study is to determine the ability of early antigen detection, isolation of RSV (+) newborns with LRTI and applied of standard infection control procedures to prevent nosocomial spread of virus from isolation rooms to the Neonatal Intensive Care Unit (NICU) where high-risk infants were under care.

\section{Subjects and Methods}

Our NICU (Regional Training and Research Hospital, Erzurum, Turkey) has a 25-patient capacity with 23 incubators and two open-bed and six mechanical ventilators. Our NICU consist of five different rooms on the same floor of the hospital. Our annual average patient number is around 1200. Approximately 200 of these are premature infants. The study was carried out prospectively between 1 October, 2010 and 31 March, 2011.

During the RSV season (October through March in Turkey [15]) all newborns who were admitted to the NICU for LRTI (Group 1) were first tested using an antigen detection kit to identify RSV (+) neonates. RSV $(+)$ neonates with LRTI (Group 1A) were isolated and segregated in a room separate from RSV (-) neonates with LRTI (Group 1B). Infants at high risk (gestational age $\leq 32$ weeks, infants with bronchopulmonary dysplasia or hemodynamically significant congenital heart disease) for RSV infection who had been hospitalized continuously since birth (Group 2) were also segregated and observed at least weekly as sentinels for nosocomial spread of RSV infection. Group 2 infants were tested weekly for evidence of RSV infection using the antigen detection kit and monitored for respiratory symptoms.

All newborns were followed in the same NICU. Standard infection control procedures (face masks, hand washing, gowns, gloves, visitor (only mother) and separation of the nursing staff) were in place throughout the study period. Each patient has dedicated instruments (e.g., stethoscopes) at their bedside. Infection control procedures were not including monitoring house staff and visitors for RSV shedding using the antigen detection kit. 
Neonates for group 1 were included in the study if there were 7 to $<30$ days of age, if they had evidence of lower respiratory tract symptoms with physical or radiographic signs and symptoms of infection such as fever, cough, tachypnea, hypoxemia (Oxygen Saturation $\left(\mathrm{SpO}_{2}\right)$ measured by Masimo Radical 7 pulse oximetry (USA) $<88 \%$ in room air [3]), retractions, nasal flaring, groaning, apnea, wheezing, rhonchi, rales, and/or infiltration on chest $\mathrm{x}$-ray. The $\mathrm{SpO}_{2}$ measurement was taken when the baby was quiet, and after suctioning to remove secretions. Measurements repeated within the first hour of admission to confirm the reading $\mathrm{SpO}_{2}$ level.

Infants were excluded if there was evidence of respiratory distress syndrome from birth, meconium aspiration, or congenital pneumonia or if they had evidence of congenital abnormalities of the respiratory tract, heart, CNS or evidence of metabolic disease that could lead to respiratory symptoms.

At admission, RSV antigens, arterial blood gas, hemogram, C-Reactive Protein (CRP), blood cultures and chest $\mathrm{x}$-rays were obtained. Patients with viral pneumonia were treated conservatively (oxygen, hydration or mechanical ventilation). Group 1B infants with bacterial pneumonia were treated with antibiotics cefotaxime and ampicillin initially. Treatments were adjusted according to culture results. Infants in group 2 were not treated with palivizumab. Mechanic ventilation requirement and duration of hospitalization were noted.

We obtained ethical committee approval and a written consent form from the families of all newborns.

RSV antigen was screened using Respi-Strip kits (Coris BioConcept, Gembloux, Belgium) following the manufacturer's recommendations. Nasopharyngeal swabs were taken, placed into tubes containing normal saline solution and agitated. Eight drops of extraction buffer solution were dripped into this mixture. The test strip was submerged into this and incubated for $15 \mathrm{~min}$ before reading. The presence of a positive control line with a positive test line was taken as a positive result. Samples could not be confirmed with PCR due to technical difficulties.

\section{Statistical Analysis}

SPSS 17 was used for statistical evaluation. Descriptive statistics were used for all the studied variables. Conformity of data obtained by measurements to normal distribution was analyzed using the Kolmogorov-Smirnov test. Data in conformity with normal distribution were analyzed using the Student $t$ test, and those not conforming using the Mann-Whitney $U$ test. Data obtained by measurement are given as mean \pm standard deviation (min-max). Data obtained by counting are presented as numbers (\%); analyses were performed using the chi square test. $\mathrm{p}<0.05$ was regarded as statistically significant.

\section{Results}

Forty one newborns were hospitalized for LRTI during the study. All of them were screened for RSV just before hospitalization. There were 15 RSV (+) infants (Group 1A) and of those, 5 newborns were admitted from another hospital and 10 were admitted directly from the community; 26 LRTI cases (Group 1B) did not have RSV upon admission; none of the 41 neonates with LRTI had received palivizumab prophylaxis. There were no differences in demographics (Table 1), physical or laboratory findings upon admission with the exception of a higher heart rates, higher white blood cell count and higher CRP in group 1B (RSV (-)) when compared to group 1A (RSV $(+)$ ) (Table 2). Infants in group 1B were hospitalized for a mean of 10.4 days but this was not significantly different from the duration of hospitalization for group 1A infants (mean of 8.1 days) and more group $1 \mathrm{~B}$ infants required mechanical ventilation $(\mathrm{n}=6,(23 \%))$ than $1 \mathrm{~A}$ infants $(n=2,(13 \%))$. There were no deaths in either group. The 36 infants that were monitored as group 2 remained free of RSV throughout the study period. Among group 2 infants, 116 antigen tests performed over the study period. Group 2 infants monitored for respiratory symptoms and RSV antigen. No infants have extra respiratory symptoms, an increase need for oxygen or ventilator assistance during the study period.

At chest radiography, diffuse reticulonodular infiltration and hyper aeration were observed in all cases in group $1 \mathrm{~A}$, as well as linear atelectasis in one case. In group $1 \mathrm{~B}$, seven cases had diffuse reticulonodular infiltration and 19 had alveolar type infiltration. While no newborns in group 1A had blood culture positivity, three cases in group 1B have blood culture positivity (Staphylococcus hominis was grown in two cases and Staphylococcus aureus was grown in one case). There was no growth in the tracheal aspirate cultures from infants requiring mechanical ventilation.

\begin{tabular}{|c|c|c|c|}
\hline & Group 1A (n=15) & Group 1B $(n=26)$ & Group $2(n=36)$ \\
\hline \multicolumn{4}{|l|}{ Sex } \\
\hline M: n (\%) & $10(67)$ & $18(69)$ & $20(56)$ \\
\hline F: n (\%) & $5(33)$ & $8(31)$ & $16(44)$ \\
\hline \multicolumn{4}{|l|}{ Delivery type } \\
\hline SVD n (\%) & $10(67)$ & $15(58)$ & $18(50)$ \\
\hline C/S n (\%) & $5(33)$ & $11(42)$ & $18(50)$ \\
\hline \multirow{2}{*}{ Birth weight (g) } & $2846 \pm 719$ & $2990 \pm 459$ & $1430 \pm 115$ \\
\hline & $(1220-3750)$ & $(2400-4300)$ & $(1250-1650)$ \\
\hline $\begin{array}{l}\text { Gestational age } \\
\text { (weeks) }\end{array}$ & $37.2 \pm 2.9(28-40)$ & $38.2 \pm 1.3(35-41)$ & $31.1 \pm 1.2(26-32)$ \\
\hline$<35$ weeks n (\%) & $2(13)$ & $0(0)$ & $36(100)$ \\
\hline$\geq 35$ weeks n $(\%)$ & $13(87)$ & $26(100)$ & $0(0)$ \\
\hline $\begin{array}{l}\text { Postnatal age } \\
\text { (days) }\end{array}$ & $17.7 \pm 5.6(9-28)$ & $19.3 \pm 6.7(9-28)$ & $7.0 \pm 5.9(1-27)$ \\
\hline
\end{tabular}

\section{Discussion}

Table 1: Demographic characteristics of the cases.

RSV is the most common cause of pneumonia and bronchiolitis in infants and is responsible for significant morbidity and mortality in high risk groups [1,3]. RSV is a respiratory infection agent commonly seen during childhood all around the world [1,3]. RSV infection rates have been reported between $29.5 \%$ and 39\% during childhood in Turkey [16-18]. Hacımustafaoğlu et al., [18] reported that incidence of RSV in infants below 3 months old is $47.9 \%$. In Brazil, in the same age group RSV incidence was reported as 50\% [19]. In our study, RSV related LRTI was $37 \%$. This high level indicates that especially hospitalized newborns in RSV risk groups have severe potential danger for RSV infection in the NICU.

Diagnosis of pneumonia was made by symptoms and characteristic findings on $x$-ray. All of the group 1 infants met these criteria. Fifteen newborns with LRTI were RSV positive and 26 were RSV negative in our study. In all cases with LRTI, $\mathrm{SpO}_{2}$ levels of were under level of $88 \%$, regarded as hypoxemia and all of them were have various LRTI symptoms. Newborns with RSV negative LRTI had higher heart rate, white blood cell counts, CRP values and mechanical ventilation requirement than RSV positive patients. These conditions 


\begin{tabular}{|l|c|c|}
\hline & Group 1A (n=15) & Group 1B (n=26) \\
\hline Respiratory Rate (mins) & $68 \pm 4.7(62-76)$ & $69.9 \pm 8.2(56-96)$ \\
\hline Heart Rate (mins) & $147.3 \pm 25.5(96-186)^{\mathrm{a}}$ & $164 \pm 17(96-182)^{\mathrm{b}}$ \\
\hline Cough, $\mathrm{n}(\%)$ & $15(100)$ & $26(100)$ \\
\hline Rales, $\mathrm{n}(\%)$ & $15(100)$ & $26(100)$ \\
\hline Retraction, $\mathrm{n}(\%)$ & $9(60)$ & $18(69)$ \\
\hline Fever, $\mathrm{n}(\%)$ & $9(60)$ & $17(65)$ \\
\hline Rhonchus, $\mathrm{n}(\%)$ & $7(47)$ & $12(46)$ \\
\hline Groaning, $\mathrm{n}(\%)$ & $6(40)$ & $12(46)$ \\
\hline Wheezing, $\mathrm{n}(\%)$ & $6(40)$ & $5(19)$ \\
\hline Nasal flaring, $\mathrm{n}(\%)$ & $2(13)$ & $5(19)$ \\
\hline Apnea, $\mathrm{n}(\%)$ & $1(7)$ & $1(4)$ \\
\hline $\begin{array}{l}\text { White Blood Cell }\left(\mathrm{x} 10^{3} /\right. \\
\text { uL) }\end{array}$ & $8.9 \pm 2.9(4.8-16.0)^{\mathrm{c}}$ & $12.2 \pm 4.5(5.8-29.0)^{\mathrm{d}}$ \\
\hline Blood gas pH & $7.34 \pm 0.09(7.1-7.38)$ & $7.33 \pm 0.06(7.2-7.4)$ \\
\hline pCO 2 & $45.7 \pm 7.6(41.3-57.8)$ & $49.1 \pm 10.3(37.6-62.8)$ \\
\hline pO 2 & $55.9 \pm 7.2(46.4-69.6)$ & $55.7 \pm 5.4(48.3-65)$ \\
\hline $\mathrm{SpO}_{2}(\%)$ & $81.9 \pm 3.7(76-86)$ & $82.2 \pm 4.2(72-87)$ \\
\hline $\mathrm{CRP}^{\mathrm{d}}(\mathrm{mg} / \mathrm{L})^{*}$ & $3.6 \pm 2.1(2.8-8.6)^{\mathrm{e}}$ & $17.2 \pm 24.9(2.9-106.4)^{\mathrm{f}}$ \\
\hline Hospitalization duration & $8.1 \pm 3.9(6-21)$ & $10.4 \pm 2.9(4-21)$ \\
\hline $\begin{array}{l}\text { Requirement for Mechani- } \\
\text { cal Ventilation, } \mathrm{n}(\%)\end{array}$ & $2(13)$ & $6(23)$ \\
\hline
\end{tabular}

Table 2: Clinical course, physical examination and laboratory findings of newborns with LRTI.

$P<0.001$ : e-f; $p<0.05: a-b, c-d$

*Normal range: (0-8 mg/L)

LRTI: Lower Respiratory Tract Infection

may be connected with severity of the illness or most of the group $1 \mathrm{~B}$ patients having pneumonia caused by bacterial micro-organisms. Bacterial micro-organisms were grown in blood cultures in only three cases.

Protection with active immunization against RSV is not yet available. There is very little information on the management of outbreaks of nosocomial RSV infection [20]. At present, protection of newborn infants in the RSV risk group involves passive immunization (palivizumab) and standard infection control procedures. Palivizumab prophylaxis in NICU outbreaks is not standardized yet. It is only approved to use for high risk infants after discharge in RSV seasons. Palivizumab prophylaxis has been reported to prevent RSV infection in healthy newborn infants in the risk groups when they are in the NICU during RSV outbreaks [5-14]. Abadesso et al., [7] concluded that standard infection control procedures might have been insufficient in the prevention of RSV outbreaks in the NICU and that the use of palivizumab might have contributed to the prevention of such outbreaks. Dizdar et al., [8] also reported that RSV outbreaks in the NICU could be successfully prevented by standard infection control procedures and palivizumab prophylaxis. Kurz et al., [9] reported that administration of palivizumab to risk groups when hospitalized in the NICU and standard infection control procedures prevented an outbreak after determining RSV infection in a premature neonate with a gestational age of 28 weeks and birth weight of $965 \mathrm{~g}$ in the NICU. Cox et al., [11] reported that standard infection control procedures failed to prevent spread of RSV and there were no further cases with administration of palivizumab prophylaxis to high-risk preterm infants. Palivizumab prophylaxis and standard infection control procedures were applied in these studies and RSV outbreaks successfully prevented.
In our study, there was no nosocomial spread of RSV in our study due to the screening for RSV infection of all newborns with LRTI just before hospitalization, isolation and attention to infection control measurement. Group 2 infants have no extra respiratory symptoms, an increase need for oxygen or ventilator assistance during the study period. Group 2 patient were not immunized with palivizumab before being hospitalized.

Although we were able to prevent any potential outbreak with screening of the patients with LRTI for RSV firm infection control procedures, these might be insufficient in preventing all outbreaks. Because infected patients, health care workers and all instruments used for patients may be carrier of RSV. RSV can infect infants via direct contact through droplet inhalation or contaminated surfaces. It can survive for up to seven hour on surfaces, gloves, tissues and clothes and for $30 \mathrm{~min}$ on the skin; infected individuals can spread the virus in their surrounding areas for 2-3 weeks [21].

The RSV Respi-Strip assay can be used as a rapid method for detecting RSV in samples from pediatric patients. Gregson et al., [22] reported that the sensitivity and specificity of the RSV Respi-Strip were $92 \%$ and $98 \%$, respectively, with a diagnostic efficiency of $95 \%$. The limitation of this study is that Respi-Strip RSV assay could not be confirmed with Polymerase Chain Reaction (PCR). But samples could not be confirmed with PCR due to technical difficulties.

There are multiple viruses that have been implicated in the etiology of lower respiratory tract infection among infants, and all of these viruses might represent a severe potential danger for newborns (e.g., adenovirus, metapneumovirus, influenza etc.). Again, these viruses could not be investigated due to technical difficulties. Nasopharyngeal swabs may also be tested for bacterial (super) infection. There was no worsening in the group 1 patients during follow up. Therefore, nasopharyngeal swabs were not taken.

In conclusion, this study shows that the rate of RSV infection in newborn infants is quite high, at 37\%, screening of RSV in newborns with LRTI just before hospitalization, isolation of infants with RSV and strict adherence to infection control procedures might prevent nosocomial spread of RSV infection in the NICU that care for at risk infants even in the absence of palivizumab prophylaxis. Standard infection control procedures, isolation and RSV antigen test are simple and easily available in terms of preventing RSV outbreaks in the NICU.

\section{References}

1. Committee on Infectious Diseases (2009) From the American Academy of Pediatrics: Policy statements--Modified recommendations for use of palivizumab for prevention of respiratory syncytial virus infections. Pediatrics 124 : 1694-1701.

2. Groothuis JR, Hoopes JM, Jessie VG (2011) Prevention of serious respiratory syncytial virus-related illness. I: Disease pathogenesis and early attempts at prevention. Adv Ther 28: 91-109.

3. Nair H, Nokes DJ, Gessner BD, Dherani M, Madhi SA, et al. (2010) Global burden of acute lower respiratory infections due to respiratory syncytial virus in young children: a systematic review and meta-analysis. Lancet 375: 15451555.

4. Halasa NB, Williams JV, Wilson GJ, Walsch WF, Scaffner W, et al. (2005) Medical and economic impact of a respiratory syncytial virus outbreak in a neonatal intensive care unit. Pediatr Infect Dis J 24: 1040-1044.

5. O'Connell K, Boo TW, Keady D, Niriain U, O'Donovan D, et al. (2011) Use of palivizumab and infection control measures to control an outbreak of respiratory syncytial virus in a neonatal intensive care unit confirmed by real-time polymerase chain reaction. J Hosp Infect 77: 338-342. 
Citation: Mutlu M, Söğüt A, Kader Ş, Aslan Y (2015) Respiratory Syncytial Virus Outbreak Prevention by Screening Neonates with Respiratory Infection, Isolation and Applying Standard Infection Control Procedures. J Neonatol Clin Pediatr 2: 006.

6. Camila de A Silva, Lívio Dias, Sandra R Baltieri, Tatiane T Rodrigues, Neusa Brandolise Takagi, et al. (2012) Respiratory syncytial virus outbreak in neonatal intensive care unit: Impact of infection control measures plus palivizumab use. Antimicrob Resist Infect Control 1: 16.

7. Abadesso C, Almeida HI, Virella D, Carreiro MH, Machado MC (2004) Use of palivizumab to control an outbreak of syncytial respiratory virus in a neonata intensive care unit. J Hosp Infect 58: 38-41.

8. Dizdar EA, Aydemir C, Erdeve O, Sari FN, Oguz S, et al. (2010) Respiratory syncytial virus outbreak defined by rapid screening in a neonatal intensive care unit. J Hosp Infect 75: 292-294.

9. Kurz H, Herbich K, Janata O, Sterniste W, Bauer K (2008) Experience with the use of palivizumab together with infection control measures to preven respiratory syncytial virus outbreaks in neonatal intensive care units. J Hosp Infect 70: 246-252

10. Groothuis J, Bauman J, Malinoski F, Eggleston M (2008) Strategies for prevention of RSV nosocomial infection. J Perinatol 28: 319-323.

11. Cox RA, Rao P, Brandon-Cox C (2001) The use of palivizumab monoclonal antibody to control an outbreak of respiratory syncytial virus infection in a special care baby unit. J Hosp Infect 48: 186-192.

12. Alan S, Okulu E, Kilic A, Atasay B, Arsan S (2012) Palivizumab use during respiratory syncytial virus outbreak in the neonatal intensive care unit. J Hosp Infect 81:292-293.

13. Alan S, Cakir U, Erdeve O, Atasay B, Arsan S (2013) Palivizumab prophylaxis during respiratory syncytial virus outbreak in the neonatal intensive care unit. Turk J Pediatr 55: 237-238.
14. Öncel MY, Mutlu B, Kavurt S, Baș AY, Demirel N, et al. (2012) Respiratory syncytial virus prophylaxis in preterm infants: a cost-effectiveness study from Turkey. Turk J Pediatr 54: 344-351.

15. Hacımustafaoğlu M (2006) RSV Infections. Ankem Derg 20: 240-47.

16. Yilmaz G, Uzel N, Isik N, Baysal SU, Aslan S, et al. (1999) Viral lower respiratory tract infections in children in Istanbul, Turkey. Pediatr Infect Dis J 18: 173.

17. Kanra G, Tezcan S, Yilmaz G (2005) Turkish National Respiratory Syncytial Virus (RSV) Team. Respiratory syncytial virus epidemiology in Turkey. Turk J Pediatr 47: 303-308.

18. Hacımustafaoğlu M, Çelebi S, Bozdemir SE, Ozgür T, Ozcan I, et al. (2013) RSV frequency in children below 2 years hospitalized for lower respiratory tract infections. Turk J Pediatr 55: 130-139.

19. Pecchini R, Berezin EN, Felício MC, Passos SD, Souza MC, et al. (2008) Incidence and clinical characteristics of the infection by the respiratory syncytial virus in children admitted in Santa Casa de São Paulo Hospital. Braz J Infect Dis 12: 476-479.

20. Bont $L$ (2009) Nosocomial RSV infection control and outbreak management. Paediatr Respir Rev 10: 16-17.

21. Hall CB, Douglas RG Jr (1981) Modes of transmission of respiratory syncytial virus. J Pediatr 99: 100-103.

22. Gregson D, Lloyd T, Buchan S, Church D (2005) Comparison of the RSV respi-strip with direct fluorescent-antigen detection for diagnosis of respiratory syncytial virus infection in pediatric patients. J Clin Microbiol 43: 5782-5783. 